\title{
Antigenic evolution can drive virulence evolution
}

\author{
Repeated epidemic waves, characteristic of an antigenically evolving pathogen, can drive selection for higher \\ pathogen virulence
}

\section{Nicole Mideo and Tsukushi Kamiya}

E volution poses many challenges for controlling infectious diseases. One obstacle is the ability of pathogens to escape existing immune responses of previously infected (or vaccinated) hosts when those responses do not generate strong protection against antigenically different strains. Antigenic evolution is the reason seasonal influenza vaccines need regular updating, for example, and has been a major focus of theoretical and empirical studies (see, for example, refs. ${ }^{1,2}$ ). Another focus has been virulence evolution: understanding what shapes disease severity ${ }^{3}$ and whether its evolution could be managed ${ }^{4}$ represent basic evolutionary puzzles with huge applied relevance. The desire to be able to predict both antigenic and virulence evolution have been amplified by the ongoing COVID-19 pandemic ${ }^{5}$, yet virtually every theoretical study has focused on the evolution of one of those traits in isolation. Now, writing in Nature Ecology \& Evolution, Sasaki et al. ${ }^{6}$ explore what happens when a pathogen's antigens and virulence are allowed to evolve simultaneously.

Sasaki and colleagues develop and analyse a mathematical model of disease spread in a host population, tracking virulence and the antigenic trait of the pathogens, along with the consequences of infection-induced immunity on any future infection. One technical challenge that the authors needed to overcome was the fact that antigenic evolution results in successive epidemic waves: a host population builds up immunity, via infection (as in their model) and/or vaccination, to a particular antigenic variant and the epidemic subsides due to a lack of susceptible individuals until a new variant arises that is sufficiently antigenically different to cause re-infections. This means that the system does not reach an equilibrium where the disease is endemic, which is a requirement of most $^{3}$, though not all (for example, ref. ${ }^{7}$ ), previous mathematical analyses of virulence evolution. Sasaki and colleagues overcome this challenge by applying an approach ${ }^{8}$ that allows them to track the evolution of different 'morphs' in pathogen trait space at the same time, where each morph is a cluster of antigenically similar pathogens, defined by the mean and variance of their antigenic trait and virulence. A key result from their analysis is that successive epidemic waves can drive the evolution of higher pathogen virulence.

One way to think about this result is to consider what governs virulence evolution in simpler models that ignore antigenic evolution ${ }^{3}$. When equated with host mortality, virulence tends to be intrinsically costly for pathogens since it often ends opportunities for future transmission. However, when virulence is correlated with transmission rate (for example, faster growing pathogens are more likely to cause new infections, but also end the infection early; a common theoretical assumption ${ }^{3}$ with some empirical support ${ }^{9}$ ), there can be a benefit to increasing virulence. In some cases, models predict that these contrasting effects - increasing transmission rate but decreasing the duration of infection - lead pathogen fitness to be maximized at intermediate levels of virulence. In epidemiological terms, this level of virulence maximizes the pathogen's reproductive number, $R_{0}$. In terms of pathogen fitness, virulence evolves to the point where the costs to infection duration are balanced by the benefits to transmission rate. When antigenic evolution is thrown into the mix, the ability to escape host immune responses and cause onward transmission is a nudge on the side of the benefits. In a sense, a pathogen strain with a novel antigenic background can 'absorb' the costs of a higher level of virulence.

Sasaki and colleagues find that partial cross-immunity, which arises when previous infection with a particular strain confers protection against more than just that strain, leads to antigenic evolution occurring in jumps. Larger steps in antigenic space are required to set off a new wave of infections among individuals with sufficient susceptibility. Once this susceptible pool has been unlocked, it sets up further competition for access to that pool: strains with higher transmission rates a No antigenic variation
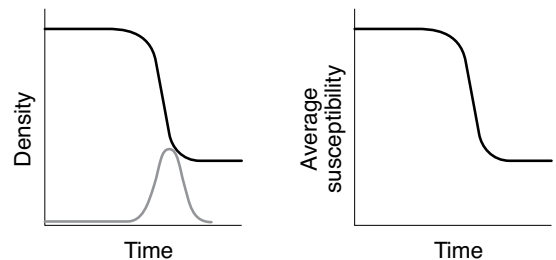

b With antigenic variation
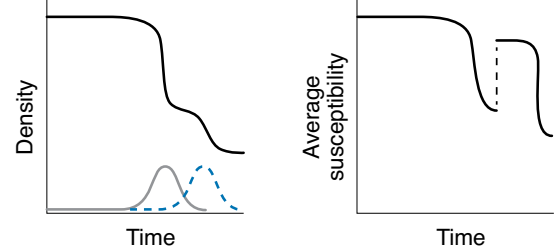

Never-infected hosts
Infected hosts
Hosts infected with new antigenic strain

Fig. 1 | How epidemiological dynamics with or without antigenic variation drive virulence evolution. In general, the strength of selection on pathogen transmission is proportional to the size of the susceptible population ${ }^{7}$ or, equivalently, the average susceptibility of the host population, as shown by Sasaki and colleagues. When transmission and virulence are correlated, selection for increased transmission gives rise to indirect selection for increased virulence. a, With no antigenic variation, the density of hosts who have never been infected (black line, left) declines over the course of an epidemic as the density of infected hosts (grey line) rises and falls. As a consequence, average susceptibility also declines through time (right), driving reductions in the strength of selection for increased transmission and, hence, increased virulence. b. With antigenic variation, the shape of decline in uninfected hosts may vary, depending on the timing of the introduction of a new antigenic strain (infections indicated by dashed blue line, left) though may quantitatively match the outcome in a. However, in the absence of strong cross-immunity, average susceptibility increases dramatically with the introduction of a new antigenic variant (right), leading to increasing selective pressure for higher transmission and thus virulence. More waves of infection driven by novel antigenic variants mean more periods of selection for high virulence. 
and, concomitantly, higher virulence will be able to exploit that pool more quickly. In a similar way, previous work has shown that early in an epidemic, selection can transiently favour high transmission rates even at the cost of higher virulence ${ }^{10}$ (in both cases, higher than values that would maximize the pathogen's $R_{0}$ ). Sasaki et al. show that antigenic evolution essentially transports the system back into the early epidemic phase, over and over, maintaining selection for these higher levels of virulence over the long term (Fig. 1).

Influenza is perhaps the archetypal pathogen that causes acute infections and elicits immune responses with partial cross-immunity. So does this work imply that the recurring, seasonal epidemics of influenza, which are at least partially a consequence of antigenic evolution, maintain selection for high levels of virulence in these viruses? Fitting with this prediction is the fact that $\mathrm{H} 3 \mathrm{~N} 2$ is the influenza subtype associated with higher hospitalization and mortality rates ${ }^{11}$ and the component of the influenza vaccine (out of four subtypes) that is updated most often due to antigenic evolution among circulating strains. As Sasaki and colleagues point out, it is hard to disentangle causality here: we might expect worse outcomes for subtypes that are more antigenically variable since on average hosts will have less effective cross-immunity, regardless of any intrinsic differences in pathogen virulence. Analyses of primary (first) infections - presumably in young children - could provide illumination if any effects of maternally transferred antibodies are sufficiently small.

A further wrinkle is that while Sasaki and colleagues assume a trade-off between transmission and virulence, other trade-offs may exist and influence evolutionary outcomes. In particular, in their model, a positive covariance between virulence and antigenicity emerges due to epidemiological dynamics, yet for some pathogens, data suggest that traits permitting immune escape simultaneously reduce replicative capacity ${ }^{12}$ (in other words, there is negative covariance between antigenic escape and a trait that is commonly assumed to underlie virulence). Thus, intrinsic biological details may further constrain the joint evolution of antigenic escape and virulence. Pathogen-specific models that capture such cellular interactions at the within-host level, along with their consequences, could provide further insight. For example, antigenic escape could exacerbate negative health outcomes due to a lack of effective immune response that would otherwise protect the host, potentially generating selection for reduced virulence to compensate. However, if simultaneous infection with different antigenic variants is common, the opposite would be expected ${ }^{13}$.

As vaccination rates against COVID-19 increase, there is understandable concern and debate about whether vaccines will drive the evolution of antigenically different viruses that escape vaccine-induced immunity ${ }^{14}$. Sasaki and colleagues' findings raise the additional alarming question of whether any antigenic evolution could subsequently drive virulence evolution. Unlike the natural immune protection they model - assumed to reduce transmissibility of an infection in a previously infected host, but not the host's initial susceptibility to infection nor the duration of infection vaccination against most diseases (COVID19 included $^{15}$ ) influences many, and perhaps all, of these outcomes. Vaccines with different clinical consequences are predicted to have differential impacts on virulence evolution including, in some cases, reducing virulence $^{16}$. Whether antigenic evolution could reverse these positive evolutionary outcomes of vaccination and exacerbate the negative ones is an important open question. The devil is likely in the detail, including the proportion of the population that is vaccinated, the amount of (existing or achievable) genetic variation in pathogen antigens and virulence, and the precise quantitative impacts of antigenic escape on the different types of protection afforded by vaccination ${ }^{17}$.

While intuiting the outcomes of eco- (or epi-) evolutionary dynamics is challenging, so too is finding a convincing natural example. Ecological processes can easily obscure the influence of evolution on the dynamics of disease spread ${ }^{18}$. However, combining longitudinal epidemiological data and evolutionary epidemiology models has helped to tease apart the role of virulence evolution on ecological dynamics in well-studied systems like myxomatosis and $\mathrm{HIV}^{19}$. Meanwhile, signatures of antigenic evolution can be detected from phylogenies: we know that pathogens like influenza with frequent antigenic escape are characterized by a 'ladder-like' structure ${ }^{20}$. Thus, a phylodynamics approach that simultaneously incorporates epidemiological and phylogenetic dynamics may offer empirical insights into the coevolution of antigenic escape and virulence, to test Sasaki and colleagues' theoretical findings.

Nicole Mideo (D) ${ }^{1 \times}$ and Tsukushi Kamiya ${ }^{2,3}$ ${ }^{1}$ Department of Ecology and Evolutionary Biology, University of Toronto, Toronto, Ontario, Canada. ${ }^{2} \mathrm{HRB}$ Clinical Research Facility, National University of Ireland, Galway, Ireland. ${ }^{3}$ Institute of Evolutionary Biology, Institute of Immunology and Infection Research, University of Edinburgh, Edinburgh, UK.

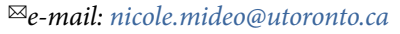

Published online: 23 December 2021 https://doi.org/10.1038/s41559-021-01600-2

\section{References}

1. Łuksza, M. \& Lässig, M. Nature 507, 57-61 (2014).

2. Koel, B. F. et al. Science 342, 976-979 (2013).

3. Alizon, S., Hurford, A., Mideo, N. \& Van Baalen, M. J. Evol. Biol. 22, 245-259 (2009).

4. Ebert, D. \& Bull, J. J. Trends Microbiol. 11, 15-20 (2003).

5. Day, T., Gandon, S., Lion, S. \& Otto, S. P. Curr. Biol. 30, R849-R857 (2020).

6. Sasaki, A., Lion, S. \& Boots, M. Nat. Ecol. Evol. https://doi. org/10.1038/s41559-021-01603-z (2021).

7. Day, T. \& Proulx, S. R. Am. Nat. 163, E40-E63 (2004).

8. Sasaki, A. \& Dieckmann, U. J. Math. Biol. 63, 601-635 (2011).

9. Fraser, C. et al. Science 343, 1243727 (2014).

10. Berngruber, T. W., Froissart, R., Choisy, M. \& Gandon, S. PLoS Pathog. 9, e1003209 (2013).

11. Pivette, M., Nicolay, N., de Lauzun, V. \& Hubert, B. Influenza Other Resp. Viruses 14, 340-348 (2020).

12. Das, S. R. et al. Proc. Natl Acad. Sci. USA 108, E1417-E1422 (2011).

13. Alizon, S. \& van Baalen, M. Am. Nat. 165, E155-E167 (2005)

14. Saad-Roy, C. M. et al. Science 372, 363-370 (2021).

15. Kissler, S. M. et al. Preprint at medRxiv https://doi.org/gh5s7f (2021).

16. Gandon, S., Mackinnon, M. J., Nee, S. \& Read, A. F. Nature 414, 751-756 (2001)

17. Miller, I. F. \& Metcalf, C. J. J. R. Soc. Interface 16, 20190642 (2019).

18. Luo, S. \& Koelle, K. Am. Nat. 181, S58-S75 (2013).

19. Bolker, B. M., Nanda, A. \& Shah, D. J. R. Soc. Interface 7, 811-822 (2010)

20. Grenfell, B. T. et al. Science 303, 327-332 (2004).

Competing interests

The authors declare no competing interests. 